\title{
OPEN MAPS OF CHAINABLE CONTINUA
}

\section{IRA ROSENHOLTZ}

ABSTRACT. It is apparently "well known" that the image of the closed unit interval under an open map is homeomorphic to the closed unit interval (see [13], [11], and [15]). In this paper, we generalize this result to chainable continua. In particular, the fact that the open continuous image of a chainable continuum is also chainable is proved, answering a question of A. Lelek (see [10]). This fact, as well as its proof, implies that the open continuous image of the pseudo-arc is also a pseudo-arc. An additional corollary (of the proof) is that a local homeomorphism of a chainable continuum is actually a homeomorphism. The proofs are all very elementary.

0. Preliminaries. All spaces are assumed to be metric. A continuum is a compact connected metric space. The statement that a continuum $X$ is chainable means that, for each positive real number $\varepsilon, X$ possesses a finite cover consisting of open subsets $C_{1}, C_{2}, \cdots, C_{n}$ of $X$, each having diameter less than $\varepsilon$, with the property that $C_{i}$ intersects $C_{j}$ if and only if $j=i-1, j=i$, or $j=i+1$. (Roughly speaking, $X$ must have open covers consisting of small sets which fit together "like a chain.") Such an open cover will be called an $\varepsilon$-chain, and the $C_{j}$ 's will be referred to as links.

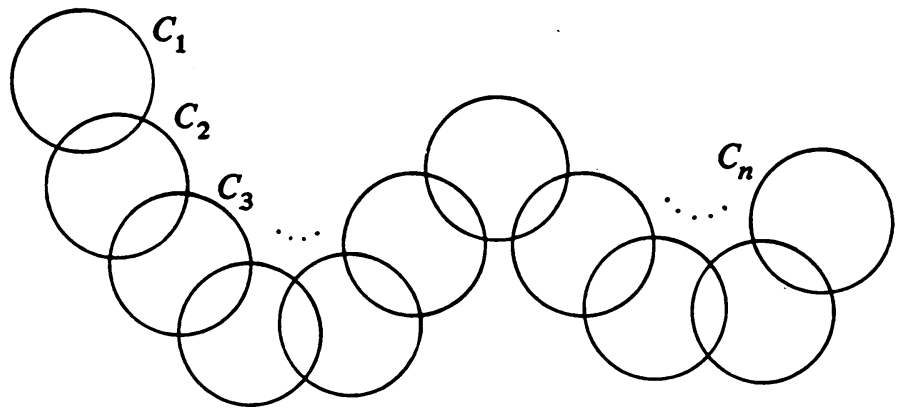

FIGURE 1

Presented to the Society, January 20, 1972 under the title Open maps of the pseudoarc and certain other metric continua; received by the editors November 27, 1972.

AMS (MOS) subject classifications (1970). Primary 65C10, 54F20.

Key words and phrases. Open maps, chainable continua, local homeomorphisms, pseudo-arc.

(c) American Mathematical Society 1974 
For example; an arc, a topologist's sine curve (i.e. the closure in the plane of $\{(x, \sin \pi / 2 x) \mid 0<x \leqq 1\}$ ), and a pseudo-arc (see [1], [14]) are chainable, while a simple closed curve and the letter " $T$ " are not.

A point $p$ of a chainable continuum $X$ is called an endpoint of $X$ if for each positive number $\varepsilon$, there is an $\varepsilon$-chain of $X$ such that only the first link contains $p$. Thus, an arc has two endpoints and a topologist's sine curve has three endpoints. The pseudo-arc has the fantastic property that it is chainable and all of its points are endpoints. This, in fact, characterizes the pseudo-arc up to homeomorphism (see [3]).

If $p$ and $q$ are points of a continuum $X$, and no proper subcontinuum of $X$ contains both $p$ and $q$, then $X$ is irreducible between $p$ and $q$. If $X$ is irreducible between some pair of its points, then $X$ is called irreducible. For example, an arc is irreducible between its endpoints, and is therefore irreducible, while a simple closed curve and the letter " $T$ " are not. It is fairly well known that chainable continua are always irreducible.

Finally, a map is a continuous function. A function from $X$ onto $Y$ is open if the image of each open subset of $X$ is an open subset of $Y$. If $X$ is compact, then a map from $X$ onto $Y$ is monotone (respectively, light) if the inverse image of each point of $Y$ is connected (respectively, totally disconnected).

1. Open maps. We now proceed to some theorems.

THEOREM 1.0. Suppose $X$ is a chainable continuum and $f$ is an open map from $X$ onto a space $Y$. Then $Y$ is also a chainable continuum.

Proof. $\quad Y$ is clearly compact and connected. Thus $Y$ is a continuum. (Remember, we are assuming metric - though Hausdorff would have been sufficient here, since $Y$ has a countable basis.)

Now suppose that $\varepsilon$ is a positive real number. We wish to show that there is an $\varepsilon$-chain of $Y$. To begin, there is a positive number $\delta$ such that if the distance between two points of $X$ is less than $\delta$, then the distance between their images in $Y$ is less than $\varepsilon$. Let $C_{1}, C_{2}, \cdots, C_{n}$ be a $\delta$-chain of $X$. Define

$$
\begin{array}{ll}
D_{1}=f\left(C_{1}\right), & D_{4}=f\left(C_{4}\right)-f\left(\bar{C}_{1} \cup \bar{C}_{2}\right), \\
D_{2}=f\left(C_{2}\right), & D_{5}=f\left(C_{5}\right)-f\left(\bar{C}_{1} \cup \bar{C}_{2} \cup \bar{C}_{3}\right), \\
D_{3}=f\left(C_{3}\right)-f\left(\bar{C}_{1}\right), & \cdot \\
& \cdot \\
& \cdot \\
& D_{j+2}=f\left(C_{j+2}\right)-f\left(\bigcup_{k=1}^{j} \bar{C}_{k}\right),
\end{array}
$$


continuing as long as the $D$ 's remain nonempty. We will show that the $D$ 's form an $\varepsilon$-chain of $Y$.

First, each $D_{k}$ is clearly open, and, by the definition of $\delta$, has diameter less than $\varepsilon$.

Next, since the $C$ 's are a $\delta$-chain of $X$, notice that, for each $k, \bar{C}_{k}$ is contained in $C_{k-1} \cup C_{k} \cup C_{k+1}$, and therefore $\bigcup_{j=1}^{k} \bar{C}_{j}$. is contained in $\bigcup_{j=1}^{k+1} C_{j}$. Hence, for each $i, D_{i}$ contains $f\left(C_{i}\right)-\bigcup_{j=1}^{i-1} f\left(C_{j}\right)$. Now since $f\left(C_{1}\right) \cup f\left(C_{2}\right) \cup \cdots \cup f\left(C_{m}\right)$ equals

$$
f\left(C_{1}\right) \cup\left(f\left(C_{2}\right)-f\left(C_{1}\right)\right) \cup \cdots \cup\left(f\left(C_{m}\right)-\bigcup_{j=1}^{m-1} f\left(C_{j}\right)\right),
$$

this is contained in $D_{1} \cup D_{2} \cup \cdots \cup D_{m}$. Therefore, since $f$ is assumed to to be onto, this implies that the $D$ 's cover $Y$.

Finally, it is clear that $D_{j}$ does not intersect $D_{k}$ if $j$ and $k$ differ by more than one. Since we already know that $Y$ is connected and the $D$ 's cover $Y$, this also implies that, for each $k, D_{k}$ intersects $D_{k+1}$. This completes the proof.

COROLlary 1.1. A nondegenerate open continuous image of an arc is an arc.

Proof. Using the fact that chainable continua are irreducible, it is easy to see that the only nondegenerate locally connected (or even pathconnected) chainable continuum is an arc.

COROLLARY 1:2. If $f$ is an open map from the chainable continuum. $X$ onto $Y$, then the image of each endpoint of $X$ is an endpoint of $Y$.

Proof. This is immediate from the proof of Theorem 1.0.

THEOREM 1.3. A nondegenerate open continuous image of the pseudoarc is again the pseudo-arc.

Proof. This follows from Corollary 1.2 and the fact that the pseudoarc is the only nondegenerate chainable continuum, each point of which is an endpoint [3].

REMARK. A person might conceivably try to simplify the proof of the fact that the pseudo-arc is homogeneous by getting an open map of the pseudo-arc onto something homogeneous (say, a simple closed curve) and having homogeneous point inverses (say, Cantor sets)-see [4]. Theorem 1.3 implies that any such attempt is doomed to failure.

Question 1. Theorem 1.0 answers a question raised by A. Lelek in his paper Some problems concerning curves [10]. In the same paper he also raises the related question: "Is the confluent image of a chainable continuum also chainable?" (The statement that the map $f$ from $A$ onto $B$ 
is confluent means that if $K$ is a continuum in $B$, then each component of $f^{-1}(K)$ maps onto all of $K$ under $f$.) By a theorem of Whyburn (see [15]), open maps defined on compact spaces are confluent, so an affirmative answer to this question would, of course, generalize Theorem 1.0. The author does not as yet know the answer to this question. McLean has some nice results in this direction (see [12]).

2. Local homeomorphisms. If $f$ is a map from $X$ onto $Y$, then the statement that $f$ is a local homeomorphism means that each point of $X$ lies in an open set which $f$ sends homeomorphically onto an open subset of $Y$. (In particular, a local homeomorphism is an open map.)

We prove the following aesthetically pleasing result.

THEOREM 2.0. Suppose that $X$ is a chainable continuum and that the map $f$ from $X$ onto a space $Y$ is a local homeomorphism. Then $f$ is actually $a$ homeomorphism.

Proof. Since $f$ is a local homeomorphism and $X$ is compact, there is a positive number $\delta$ such that if $C$ is a set of diameter less than $\delta$, then $f$ restricted to $C$ is a homeomorphism.

Let $C_{1}, C_{2}, \cdots, C_{n}$ be a $\delta / 3$-chain of $X$, and let $D_{1}, D_{2}, \cdots, D_{m}$ be the corresponding chain of $Y$ as constructed in the proof of Theorem 1.0. Recall that, for each $j, D_{j}$ is contained in $f\left(C_{j}\right)$. Thus, since the restriction $f \mid C_{j}$ is a homeomorphism, the function $\left(f \mid C_{j}\right)^{-1}: D_{j} \rightarrow C_{j}$ is a well-defined map. In addition, if $y \in D_{j} \cap D_{j+1}$, then $y \in f\left(C_{j}\right) \cap f\left(C_{j+1}\right)$, so, since $f \mid C_{j} \cup C_{j+1}$ is a homeomorphism, $\left(f \mid C_{j}\right)^{-1}(y)=\left(f \mid C_{j+1}\right)^{-1}(y)$. Thus, the function $g: Y \rightarrow X$ defined by $g \mid D_{j}=\left(f \mid C_{j}\right)^{-1}$ is well-defined and continuous. Since $f(g(y))=y$ for $y \in Y, g$ is one-to-one. By the continuity of $f, g$ is an open map, so it is actually onto, and therefore a homeomorphism onto $X$. But $f$ is its inverse, so $f$ is a homeomorphism.

REMARK. This is a "now you see it, now you don't" proof. The link $D_{j}$ appears to have part of $f\left(C_{j}\right)$ removed, but it actually did not. (Shades of Houdini!) When I first tried to prove this theorem, I started with very small chains of $X$, but I could not see how to prevent the images of the links from "coming back" and haunting me. Even having "seen" the trick, I still do not understand why it works. Here are some related questions.

Question 2. Open maps from one continuum to another are local homeomorphisms if, and only if, they are exactly $n$-to- 1 , for some positive integer $n$. Are there any exactly $n$-to- 1 maps from one chainable continuum to another-much less open ones? (Somewhat surprisingly, for $n$ greater than 2 , there exist exactly $n$-to- 1 maps from an arc onto a simple closed curve.) See [6], [8], and [9]. 
Question 3. Using Hamilton's result [7] it is possible to show, even more easily than in the proof of Theorem 2.0, that there are no exactly 2-to-1 open maps (hence local homeomorphisms) defined on any chainable continuum. Are there any exactly 2-to-1 maps from any chainable continuum to a metric space, at all? There are no exactly 2-to-1 maps of the arc. See [8].

Question 4. In what ways can Theorem 2.0 be generalized? For example, is a local homeomorphism defined on a plane continuum which does not separate the plane, necessarily a homeomorphism? For a start, see [5].

Theorem 2.0 implies its own generalization.

Corollary 2.1. Suppose $f$ is an open map from $X$ onto $Y$, where $X$ is chainable. And suppose, furthermore, that $n$ is a positive integer such that for each $y \in Y, f^{-1}(y)$ has exactly $n$ components. Then $n=1$-that is, $f$ is monotone.

PrOOF. By Whyburn's "factorization theorem" (see [15]), the open map $f$ can be factored "uniquely" into the form $f(x)=z(m(x))$, where the map $m: X \rightarrow Z$ is monotone and $z: Z \rightarrow Y$ is light open. Now use Bing's theorem that monotone images of chainable continua are chainable [2], the fact that an exactly $n$-to- 1 open map is a local homeomorphism, and Theorem 2.0.

3. Some examples. After considering elementary examples, one might be tempted to conjecture that the open continuous image of a chainable continuum is homeomorphic to a subcontinuum of the domain. That this is false is "shown" by the next example.

EXAMPLE 3.0. Let $Q$ be the "continuous arc of pseudo-arcs" described in Bing and Jones' paper [4]. Then $Q$ is a chainable continuum, and there is an open map $q$ from $Q$ onto the closed unit interval $[0,1]$ so that the inverse image under $q$ of each point is a pseudo-arc. However, $Q$ contains no arc.

The statement that a function $f$ is one-to-one at $p$ means that $p$ is a point in the domain of $f$ and $f^{-1} f(p)=\{p\}$. In many cases, it appears that, for an open map of a chainable continuum, being one-to-one at an endpoint is sufficient to guarantee that it is a homeomorphism. Recalling the proof of Theorem 1.0, we see that if the map $f$ is one-to-one at the endpoint $p$, and if we chain the continuum with $p$ in the last link, then the construction is forced to go "all the way." But again the appearance is not the fact, as the next two examples show.

EXAMPLE 3.1. Let $Q$ and $q$ be as in Example 3.0. Let $Q^{*}$ be the space obtained by smashing the end pseudo-arcs $\left(q^{-1}(0)\right.$ and $\left.q^{-1}(1)\right)$ to points, 
and let $f$ denote the natural projection of $Q$ onto $Q^{*} \cdot f$ is monotone, so $Q^{*}$ is chainable.

Let $g$ be the map from $Q^{*}$ to the interval $[0,1]$, gotten by "finishing the job that $f$ started"-i.e. $g$ smashes the rest of the pseudo-arcs to points.

That $g$ is an open map is easily verified, and $g$ is one-to-one at both endpoints $\left(f\left(q^{-1}(0)\right)\right.$ and $\left.f\left(q^{-1}(1)\right)\right)$.

However, clearly $g$ is not a homeomorphism.

EXAMPLE 3.2. There exist nontrivial period two homeomorphisms of the pseudo-arc. These induce light (in fact at most 2-to-1) open maps of the pseudo-arc which must be one-to-one at an endpoint, but which are not homeomorphisms.

In our last example, we show that a chainable continuum can have uncountably many nonhomeomorphic open images.

ExAMPLE 3.3. Let $Q$ and $q$ be as in Examples 3.0 and 3.1. Let $Q^{* *}$ be the chainable continuum obtained by smashing each of the pseudo-arcs $q^{-1}(0), q^{-1}(1), q^{-1}\left(\frac{1}{2}\right), q^{-1}\left(\frac{1}{3}\right), q^{-1}\left(\frac{1}{4}\right), \cdots$ to points. Let $S$ be a subset of the positive integers. For those $n$ belonging to $S$ (and only those), squash each pseudo-arc of $Q^{* *}$ "between $1 / 2 n$ and $1 /(2 n-1)$ " to points. This technique yields open maps of $Q^{* *}$, which are seen to have nonhomeomorphic images for distinct subsets of the positive integers.

ADDED IN PROOF. The author has found an example of a chainable continuum which admits an exactly 2-to-1 map, thus answering Question 3.

\section{BIBLIOGRAPHY}

1. R. H. Bing, A homogeneous indecomposable plane continuum, Duke Math. J. 15 (1948), 729-742. MR 10, 261.

2. - Concerning hereditarily indecomposable continua, Pacific J. Math. 1 (1951), 43-51. MR 13, 265.

3. - Snake-like continua, Duke Math. J. 18 (1951), 653-663. MR 13, 265.

4. R. H. Bing and F. B. Jones, Another homogeneous plane continuum, Trans. Amer. Math. Soc. 90 (1959), 171-192. MR 20 \#7251.

5. Samuel Eilenberg, Sur quelques propriétés des transformations localement homéomorphes, Fund. Math. 24 (1935), 35-42.

6. Paul Gilbert, n-to-one mappings of linear graphs, Duke Math. J. 9 (1942), 475-486. MR 4, 88.

7. O. H. Hamilton, $A$ fixed point theorem for pseudo-arcs and certain other metric continua, Proc. Amer. Math. Soc. 2 (1951), 173-174. MR 12, 627.

8. O. G. Harrold, Jr. The non-existence of a certain type of continuous transformation, Duke Math. J. 5 (1939), 789-793. MR 1, 223.

9. - Exactly $(k, 1)$ transformations on connected linear graphs, Amer. J. Math. 62 (1940), 823-834. MR 2, 75.

10. A. Lelek, Some problems concerning curves, Colloq. Math. 23 (1971), 93-98.

11. L. F. McAuley, Open mappings and open problems, Topology Conference (Arizona State Univ., Tempe, Ariz., 1967), Arizona State Univ., Tempe, Ariz., 1968, pp. 184-202. MR 39 \#2134. 
12. T. Bruce McLean, Confluent images of tree-like curces are tree-like, Duke Math. J. 39 (1972), 465-473.

13. S. S. Mitra, Continuous open maps on the unit intertal, Amer. Math. Monthly 76 (1969), 817-818.

14. E. E. Moise, An indecomposable plane continuum which is homeomorphic to each of its nondegenerate subcontinua, Trans. Amer. Math. Soc. 63 (1948), 581-594. MR 10, 56.

15. G. T. Whyburn, Analytic topology, Amer. Math. Soc. Collog. Publ., vol. 28, Amer. Math. Soc., Providence, R.I., 1942, 1963 ed. MR 4, 86.

Department of Mathematics; Grinnell College, Grinnell, Iowa 50112

Current address: Department of Mathematics, University of Wyoming, Laramie, Wyoming 82070 